\title{
An Updated Systematic Review of Quantitative Studies Assessing Anxiety, Depression, Fear of Cancer Recurrence or Psychological Distress in Testicular Cancer Survivors
}

\author{
Orlando Rincones (ID) \\ Allan 'Ben' Smith (ID)' \\ Sayeda Naher ${ }^{2}$ \\ Rebecca Mercieca- \\ Bebber (D) $^{2}$ \\ Martin Stockler $\mathbb{D}^{2}$ \\ 'Centre for Oncology Education and \\ Research Translation (CONCERT), \\ Ingham Institute for Applied Medical \\ Research \& University of New South \\ Wales, Liverpool, New South Wales, \\ Australia; ${ }^{2} \mathrm{NHMRC}$ Clinical Trials \\ Centre, The University of Sydney, \\ Camperdown, New South Wales, \\ Australia
}

Purpose: A diagnosis of testicular cancer (TC) at a relatively young age can have a dramatic impact on the psychological well-being of those affected. The aim of this review was to synthesize recent evidence to provide an updated account of the prevalence, severity and correlates of anxiety, depression, fear of cancer recurrence (FCR) and distress in TC survivors.

Patients and Methods: A systematic literature review was conducted from September 2017 until June 2020 using electronic databases including Embase, MEDLINE, PsycINFO, Scopus and Web of Science. Study eligibility and quality were independently assessed by two reviewers. Narrative synthesis was used to depict the severity (mean/median scores), prevalence (proportions above standard clinical thresholds) and correlates of study outcomes.

Results: A total of 988 articles were identified for screening after duplicate removal. Fiftysix full-text articles were screened, and eight articles met the inclusion criteria. The reported prevalence of the outcomes varied across studies (clinical levels of anxiety ranged from $6.9 \%$ to $21.1 \%$, depression varied from $4.7 \%$ to $7 \%$, distress was found between $25 \%$ and $41.4 \%$, prevalence of FCR was not reported). Few studies compared TC survivors with other populations. Correlates of poorer psychological outcomes included younger age, relationship status, employment status, poorer sexual functioning, impaired masculinity and coping strategies

Conclusion: Anxiety seems to be the most common issue for TC survivors. Men who are single or unemployed appear most at risk of poorer psychological outcomes, which seem associated with impaired masculinity and sexual function. More research is needed to identify TC survivors most likely to need one of the increasing number of psychological interventions being developed for TC survivors.

Keywords: anxiety, cancer survivors, depression, distress, fear of cancer recurrence, testicular neoplasms

\section{Introduction}

Testicular cancer (TC) is considered the most prevalent cancer type in young men, ${ }^{1}$ with more than 71,000 new cases in 2018 worldwide. ${ }^{2}$ Low mortality rates $(<10 \%)$ and good prognosis are the results of the highly effective main treatment options, including orchidectomy, radiotherapy and cisplatin-based chemotherapy. ${ }^{1}$ The low mortality rate means that there is a growing number of TC survivors. 
Living after TC represents a challenge in the physical, social and emotional domains. ${ }^{3}$ TC survivors have to be followed-up on a long-term basis regarding fertility and the potential of developing second cancers, hypogonadism, sexual dysfunction, cardiovascular disease, metabolic syndrome, ongoing fatigue and other concerns. ${ }^{1,4}$ The burden and risk of treatment side effects are generally higher for those who received chemotherapy. ${ }^{4}$

TC survivors are often concerned about sexuality, fertility, body image and male identity ${ }^{5-7}$ which can impact survivor's quality of life. For instance, a recent study found that at least $26 \%$ of TC patients experienced sexual dysfunction and $28 \%$ had significant reproductive concerns. ${ }^{8}$ Occupational and career-related issues also arise from the TC experience, ${ }^{7}$ causing several challenges to this population of young to middle-aged men. The adjustment to the cancer experience can also be impacted by a number of variables, including having children, and marital and employment status. ${ }^{5,6}$ Interestingly, a study showed that at least $20 \%$ of TC survivors needed psychological support due to the cancer experience even after 7-10 years post-treatment. ${ }^{9}$

Distress, anxiety and depression in TC survivors have been studied in the last few decades. The psychological impact and experience of TC diagnosis might differ from the impact and experience in other cancer types, especially those in older populations. Studies that have explored distress among TC survivors have found that participants with an experience of TC experience similar levels of distress compared to the general population or other cancer populations. ${ }^{10}$ However, TC survivors who are not employed, have lower educational status and are single are at higher risk of elevated distress. ${ }^{11}$ Another study has found an association between distress and reduced quality of life. ${ }^{12}$ In addition, chemotherapy side effects related to the cognitive function seem to be associated with higher distress. ${ }^{13}$

Our previous review found that TC survivors report higher prevalence and severity of anxiety than in the general population. ${ }^{10}$ There is also evidence that the younger the patient, the higher the risk of experiencing clinical levels of anxiety. ${ }^{14}$ Research has also revealed that higher cancer-related fatigue is associated with higher levels of anxiety in TC survivors. ${ }^{15}$

Depression has been linked to the diagnosis and posttreatment phases of TC. ${ }^{16,17}$ Our previous review found that most studies did not report higher levels of depression among TC survivors than the general population.
However, TC literature has reported various rates of depression. For instance, an Australian study found clinical levels of depression in $20 \%{ }^{16}$ of the sample while a study in the United States (California) found this outcome in $34 \% .{ }^{18}$ In contrast, a Serbian study found that $12 \%$ of TC survivors experienced mild or moderate depression. ${ }^{19}$ The National Comprehensive Cancer Network $^{20}$ indicated that there is no sufficient evidence to argue that TC survivors experience higher levels of depression than the general population.

Our previous review reported that 1 in 3 TC survivors suffer from fear of cancer recurrence (FCR) ${ }^{10}$ based on evidence from only six studies. There has been growing recognition and investigation of FCR in TC survivors, but recent studies including different samples and measures have produced mixed results regarding the prevalence of FCR among TC survivors. Studies have reported between $37 \%^{21}$ to $58 \%{ }^{22}$ of $\mathrm{TC}$ survivors experiencing abovethreshold levels of FCR. Another study ${ }^{23}$ found out that around $20 \%$ of young adult cancer survivors expressed being worried about cancer recurrence and getting another cancer. A recent systematic review found that FCR prevalence varied from $31 \%$ to $85 \%$ in adolescent and young adult cancer survivors. ${ }^{24}$

In brief, findings to date regarding distress, anxiety, depression and FCR in TC survivors are mixed. Several large studies have been published in the field since the last review and there is a pressing need to identify TC survivors who are in need of psychological support and may benefit from the growing number of psychological interventions for TC survivors. ${ }^{22,25}$ A companion review looks at summarising quality of life data concerning the physical impact of various treatments for testicular cancer. This article aims to update our previous systematic review to provide more current insights around distress, anxiety, depression and FCR among testicular cancer survivors. The review objectives remain the same as per our previous review:

1. To determine the prevalence and severity of anxiety, depression, FCR, and distress in TC survivors (compared with the general population or other cancer survivors);

2. To identify TC survivors at risk of worse outcomes by exploring correlates of anxiety, depression, FCR, and distress, such as sociodemographic, disease and treatment received, and other psychosocial measures such as personality traits, social support, self-esteem, among others;

3 . To evaluate relationships between anxiety, depression, FCR, distress, and other variables in TC survivors. 


\section{Patients and Methods}

A systematic review of literature published since October 2017 (search date of the previous review) was conducted according to Preferred Reporting Items of Systematic reviews and MetaAnalyses (PRISMA) guidelines. See Supplementary Table 1 for the PRISMA Checklist. ${ }^{26}$

\section{Search Strategy}

A total of five electronic databases were searched (Embase, MEDLINE, PsycINFO, Scopus and Web of Science) from the 1st of October 2017 to the 14th of June 2020. As this is an updated review, the same main keywords from the previous review were used including 'testicular' OR 'testis' OR 'testes', AND "cancer*" OR "neoplasm*" OR "carcino*" OR "sarcoma*" OR "malignan*" OR "tumour*" OR "tumor*” OR "metasta*", AND "anx*" OR "depress"” OR "fear of recurrence" OR "distress"” OR "stress"” OR "emotion*" OR "mental health" OR "post-traumatic stress disorder"). See a search example on Appendix 1. The search was broadened by exploding key terms or Subject Heading (MESH) terms for the above-mentioned databases. Additional ancillary search procedures were applied, including citation tracking and hand searching of reference lists of 9 articles (mostly reviews, see Appendix 2) considered relevant by authors OR and ABS.

\section{Eligibility Criteria}

Studies that met the following selection criteria were included: a) original research reporting relevant quantitative Patient Reported Outcomes (PROs) from adult patients ( $\geq 18$ years old) who completed TC treatment; b) at least $50 \%$ of the sample consisted of unilateral TC survivors who had completed treatment after 1977 (date of cisplatin-based chemotherapy implementation), or the TC survivor data is reported separately if diverse cancer survivors were included in the study; c) studies that presented quantitative patient-reported outcome (PRO) data regarding anxiety, depression, fear of cancer recurrence (FCR) or psychological distress without the influence of any intervention for a specific time point (as opposed to change scores). Studies were excluded if they were conference abstracts, not published in peer review journals, systematic or literature reviews, commentaries, policy-making documents or theoretical articles.

\section{Procedures}

The search strategy was applied individually to each database. All records were saved using EndNote and duplicates were removed. Reviewer OR screened titles and abstracts to exclude obviously irrelevant studies. The full texts of potentially relevant articles were assessed by two reviewers (OR and $\mathrm{ABS}$ ) who used the inclusion and exclusion criteria to determine eligibility. Discussions between the two reviewers took place, and a consensus was reached regarding the final articles to be included.

\section{Quality Assessment}

A modified version (reduced to 14 items) of the quality of health care intervention studies checklist ${ }^{27}$ was used to appraise the quality of the studies on four different areas: internal validity and bias (6 items), reporting (5 items), external validity ( 2 items), and power ( 1 item). Each item was scored 1 point when met, or 0 if not met or unable to be determined. Scores were transformed into a $0-100$ scale based on the total number of applicable items to each article. The checklist thresholds are poor $(\leq 49)$, fair $(50-79)$ and $\operatorname{good}(\geq 80)$. Authors OR and SN assessed each article independently; discrepancies were resolved through discussions with author ABS

\section{Data Extraction and Data Analysis}

The key data collected from each article included: research location, number of recruitment centres, study design, assessment time points, comparison group (if applicable), the total number of TC survivors and response rate, participants' age, treatment received, assessed PROs, systematic review objective addressed, overall prevalence and severity of each PRO, prevalence and severity of each PRO relative to the comparison group (when the comparison group did not include TC survivors), demographic, disease and treatment correlates of each PRO, and the association between PROs. Authors OR and SN extracted half of the articles each. Both OR and SN checked each other's extraction to make sure that all the data was collected accurately and consistently.

\section{Data Analysis}

A narrative synthesis was used to depict the severity (mean/median scores) and prevalence (proportions above standard clinical thresholds) of PROs of interest. Due to the small number of articles included and the variety of measures used, a meta-analysis was deemed inappropriate.

\section{Results}

The search identified a total of 2058 records from all sources (1568 records from electronic databases, and 490 records from reference list check and citation tracking). 
See PRISMA flow diagram in Figure 1). More than half of the records were duplicates $(n=1070)$, leaving 988 articles for screening. The initial screening of titles and abstract excluded $94.3 \%$ of the articles $(n=932)$. This resulted in 56 full-text articles being screened by two authors, who after discussions determined that eight articles met inclusion criteria for the review. PRISMA Flow Diagram.

\section{Quality Assessment}

Table 1 displays the results of the study quality assessment. Total scores on the 0-100 transformed score ranged from 64 to 92 . Five articles $(62.5 \%)$ were considered of fair quality, while three $(37.5 \%)$ were good quality. No studies were excluded from analysis due to low methodological quality and all studies were allocated an equal weighting for analysis.

\section{Study Characteristics}

Three studies were conducted in Denmark, while there was one study each from the United States, Brazil, Canada, Australia and New Zealand. One study recruited participants from multiple European countries. ${ }^{28}$ Five studies (out of 8) only had one recruitment site. The most common study design was cross-sectional $(n=6)$; however, there was one longitudinal study, ${ }^{29}$ and one randomized controlled trial. ${ }^{30}$ Study assessment time points varied

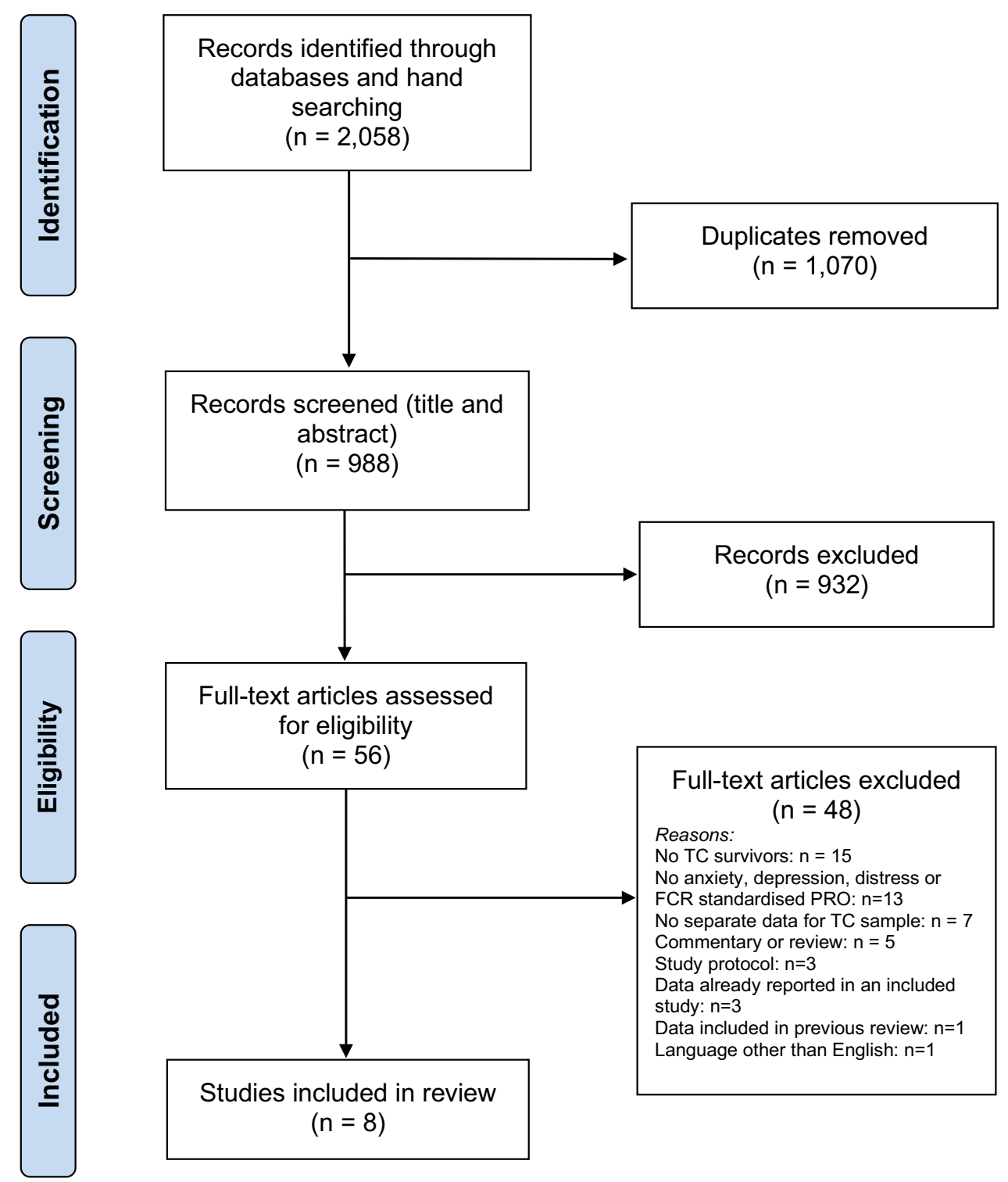

Figure I 


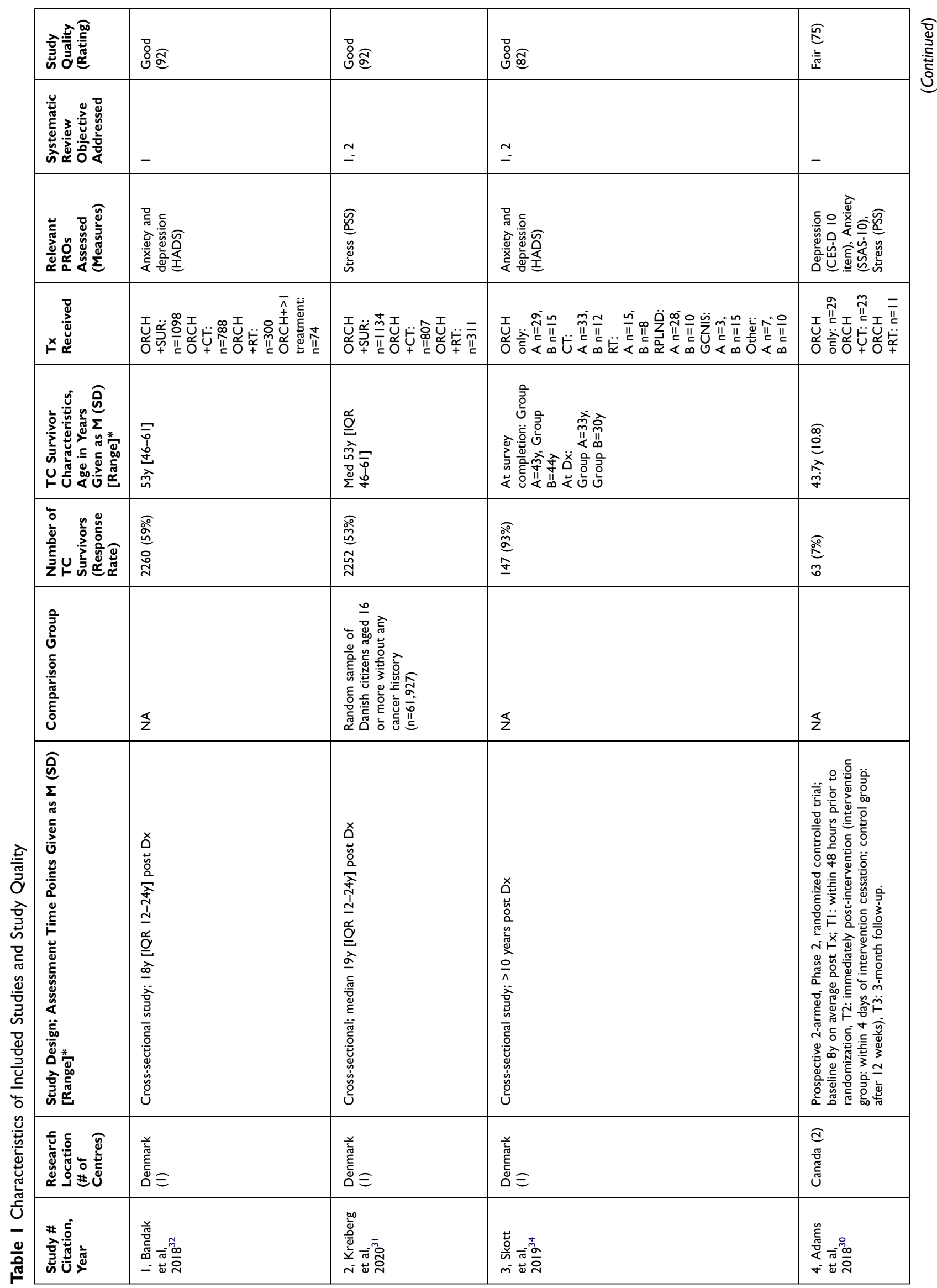




\begin{tabular}{|c|c|c|c|c|c|}
\hline 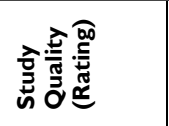 & 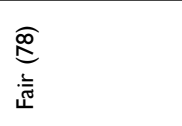 & 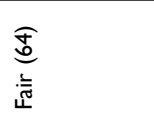 & $\underbrace{}_{\substack{\hat{0} \\
\text { 鹿 }}}$ & 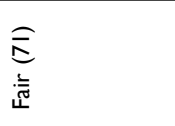 & 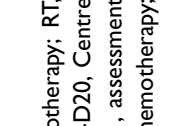 \\
\hline 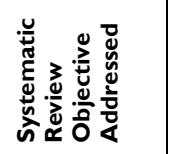 & $\stackrel{m}{=}$ & $\cong$ & $\stackrel{m}{=}$ & - & 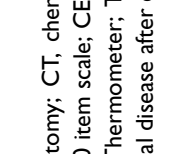 \\
\hline 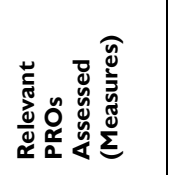 & 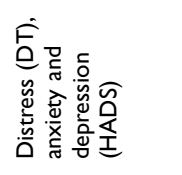 & 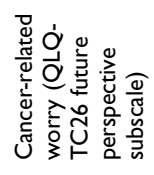 & 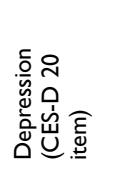 & 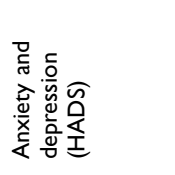 & 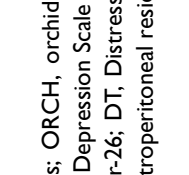 \\
\hline 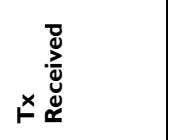 & 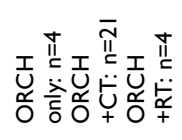 & 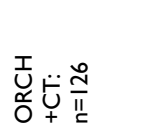 & 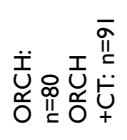 & 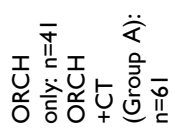 & 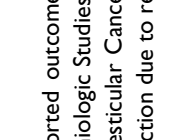 \\
\hline 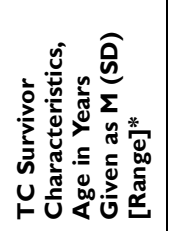 & 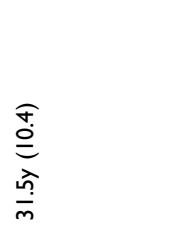 & 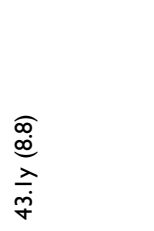 & 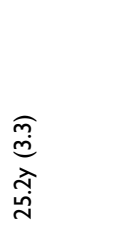 & 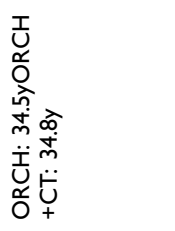 & 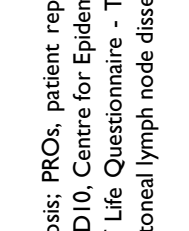 \\
\hline 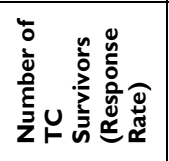 & 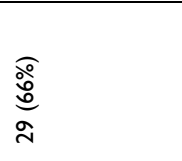 & 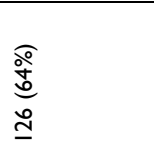 & $\frac{\underline{\underline{x}}}{\underline{\underline{\Sigma}}}$ & $\begin{array}{l}\text { o̊ } \\
\stackrel{8}{c} \\
\text { s }\end{array}$ & 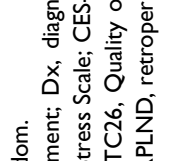 \\
\hline 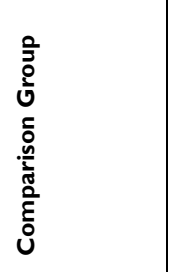 & $\mathbb{z}$ & 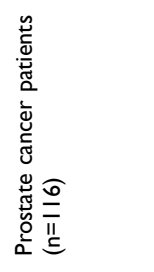 & $\underline{z}$ & $\underline{z}$ & 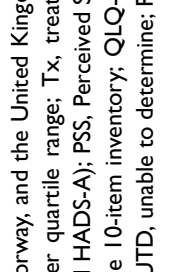 \\
\hline 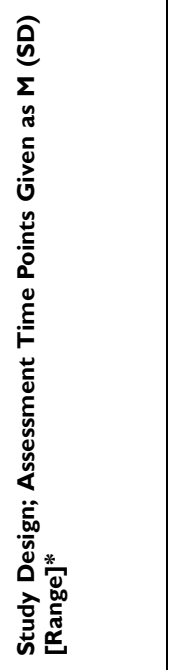 & 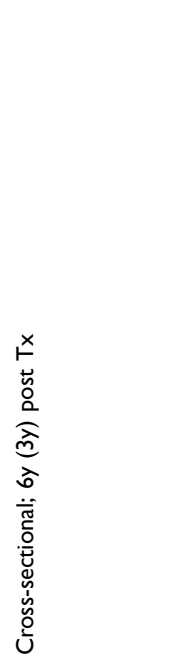 & 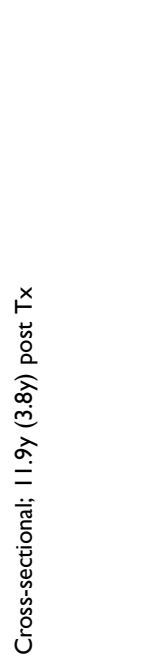 & 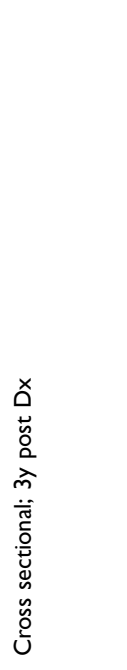 & 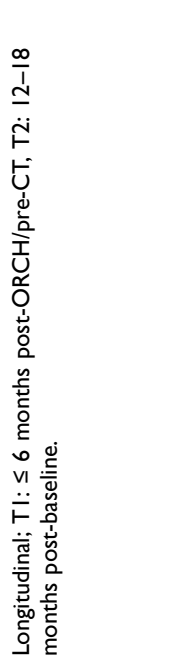 & 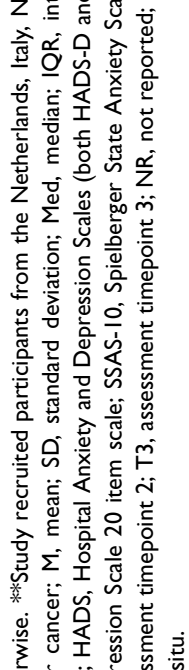 \\
\hline 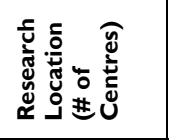 & $\begin{array}{c}\widehat{\Xi} \\
\overline{\bar{N}} \\
\overline{\tilde{D}}\end{array}$ & 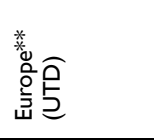 & 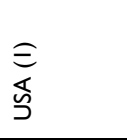 & 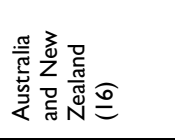 & 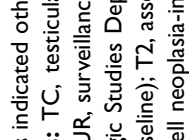 \\
\hline 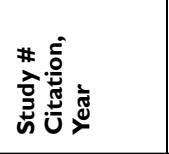 & 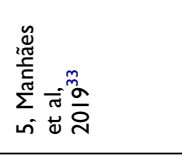 & 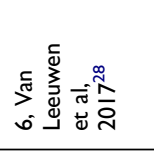 & 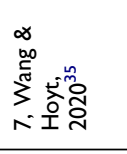 & 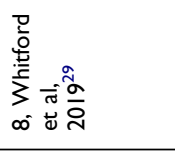 & 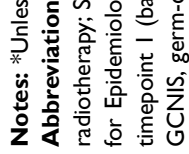 \\
\hline
\end{tabular}


from an average of 3 to 19 years post-diagnosis, or 6 months to 11.9 years post-treatment. Only two studies $^{28,31}$ had a comparison group of non-TC survivors.

Each study included between 29 and 2260 participants. The total number of participants across all studies was 2898 (one study $^{31}$ is excluded from this count as they are the same participants from another publication, ${ }^{32}$ but each study reported different outcomes). The overall participant response rate average was 58.8\% (range: 7-93\%). TC survivors were 49 years old on average (pooled mean) at the time of assessment (range: 25-61 years). Most participants were treated with orchiectomy $(n=2885 ; 99.5 \%)$, with some receiving chemotherapy $(n=1157 ; 39.9 \%)$ or radiotherapy $(n=340 ; 11.7 \%)$. All studies $(n=8)$ included TC survivors who received orchiectomy and chemotherapy. Five of these studies included survivors who also had radiotherapy. A single study ${ }^{32}$ indicated that a few participants received more than two treatments. Only one study ${ }^{28}$ exclusively included a sample of survivors who received both orchiectomy and chemotherapy. The three largest studies included all main treatment types (orchiectomy, chemotherapy, and radiotherapy), potentially reducing selection bias.

\section{Prevalence and Severity of Anxiety}

Five studies assessed anxiety (3 reported prevalence, while 3 reported severity) using the HADS-A (four studies) or the Spielberger State Anxiety Scale 10-item inventory (SSAS-10). The reported prevalence of borderline abnormal or abnormal anxiety was $6.9 \%,{ }^{33} 20 \%{ }^{32}$ and $21.1 \%{ }^{34}$ Two studies ${ }^{29,33}$ found mean HADS-A scores of 3.2 to 6.16 (scale range $0-21$ ), and another study ${ }^{30}$ reported SSAS-10 scores of 16.2 to 18.6 (scale range unclear).

\section{Correlates of Anxiety}

Anxiety was not significantly related to age ${ }^{34}$ or treatment type. $^{29}$ One study found relationships of anxiety with cognitive-emotional regulation, sexual confidence and sexual functioning. ${ }^{33}$

\section{Prevalence and Severity of Depression}

Six studies evaluated depression with either the HADS-D or the CES-D10/D20 with prevalence reported in three articles and severity scores in four articles. The prevalence of clinical levels of depression varied from $4.7 \%$ to $7 \%$ (see Table 2). Results from two studies ${ }^{29,33}$ that used the HADS-D found mean scores of 1.8 to 3.05 (scale range 0-21), while the CES-D10 scores $^{30}$ were 4.5 to 5.3 (scale range $0-30$ ), and the CES-D20 score ${ }^{35}$ was 12.99 (scale range 0-60). No comparisons with groups of non-TC survivors were reported.

\section{Correlates of Depression}

Depression was not significantly related to age $^{34}$ or treatment type. ${ }^{29}$ One study found associations of depression with cognitive-emotional regulation, sexual confidence and sexual regulation. ${ }^{33}$ Another study reported a positive and significant correlation between depression and cancer-related masculine threat, as well as a significant and negative correlation with benefit finding. ${ }^{35}$

\section{Prevalence and Severity of Fear of Cancer Recurrence (FCR)}

A single study ${ }^{28}$ reported on the severity of FCR, assessed using the QLQ-TC26 future perspective subscale, finding a mean of $77 / 100(\mathrm{SD}=24)$.

\section{Correlates of FCR}

Single men were found to report less FCR than partnered men (see Table 2).

\section{Prevalence and Severity of Distress}

Distress was assessed in three studies using the Perceived Stress Scale $(\mathrm{PSS})^{30,31}$ and the Distress Thermometer (DT). ${ }^{33}$ Prevalence was reported in two studies, and severity in three. When it comes to prevalence, the study that used the $\mathrm{DT}^{33}$ reported the highest prevalence with $41.4 \%$ $(\geq 4)$. The study that utilized the $\operatorname{PSS}^{31}$ found that $25 \%$ of their sample had scores $\geq 16$. Mean PSS scores ranged from 6.3 to 9.1 on a $0-40$ scale range. DT mean score was 2.8 (low distress) within the $0-10$ range.

\section{Correlates of Distress}

One study ${ }^{31}$ found that TC survivors experience a higher severity of distress than the general male population in a multivariable-adjusted analysis regardless of treatment received. The same study found that high distress is associated with being single, unemployed, and reporting poor self-rated fitness. ${ }^{31}$ These authors also found that TC survivors had a higher prevalence of distress than the general men population until the age of 70 (after this age, the general population becomes the more distressed). ${ }^{31}$ Distress was also found to be related to CognitiveEmotional Regulation $(\mathrm{R} 2=37 \%)$ in one study. ${ }^{33}$ 


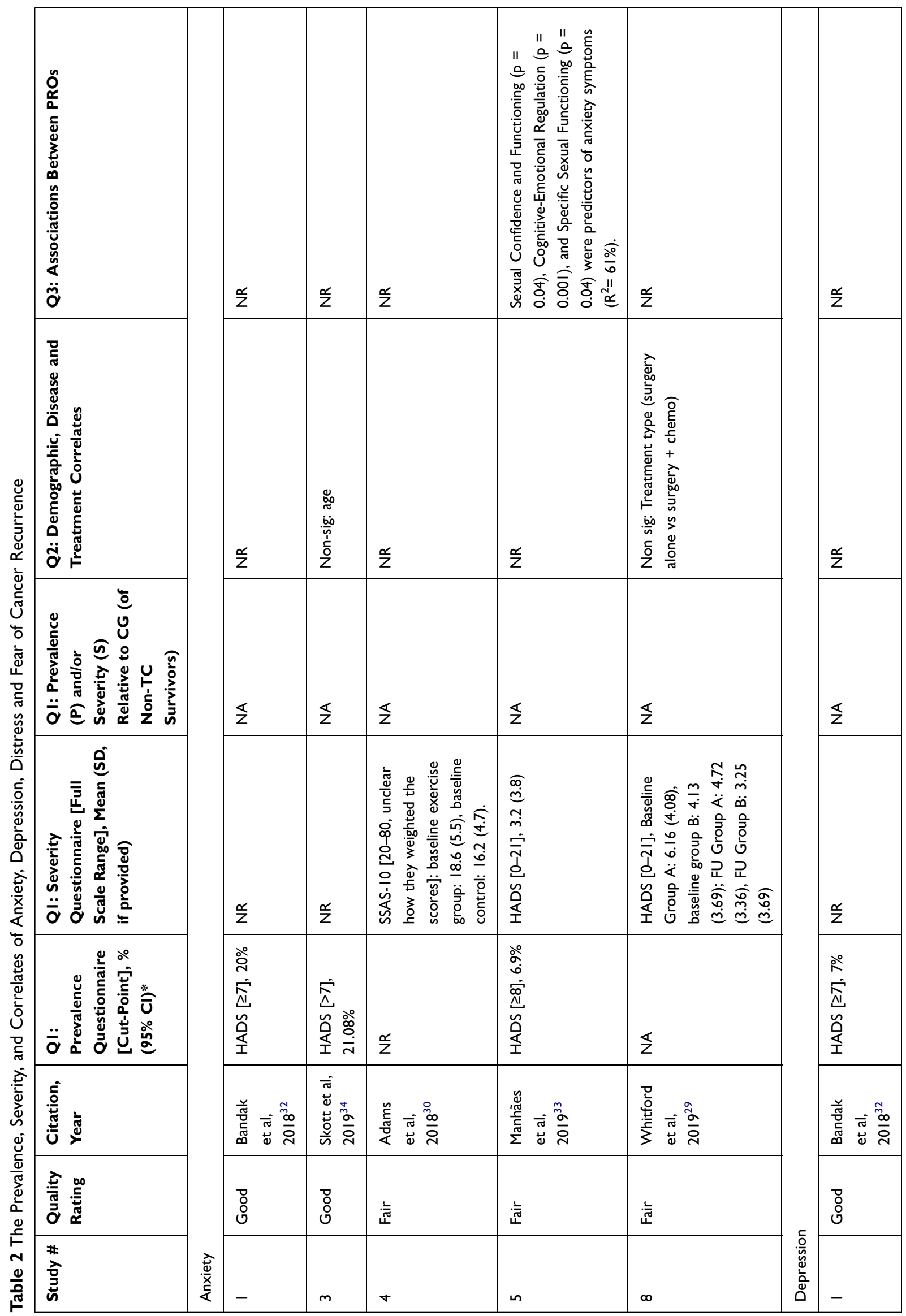




\begin{tabular}{|c|c|c|c|c|c|c|}
\hline$\frac{o}{z}$ & $\frac{o}{z}$ & 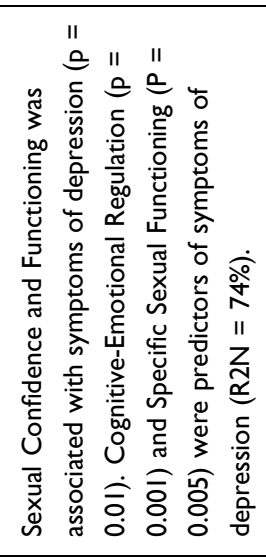 & 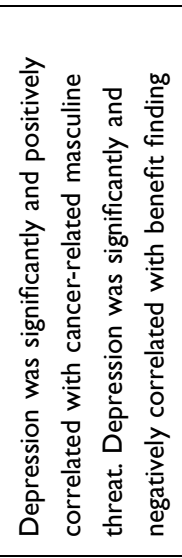 & $\stackrel{\alpha}{z}$ & & $\stackrel{\alpha}{z}$ \\
\hline 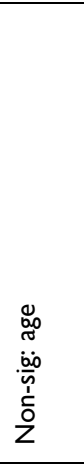 & $\stackrel{\alpha}{z}$ & $\S$ & $\frac{\alpha}{z}$ & 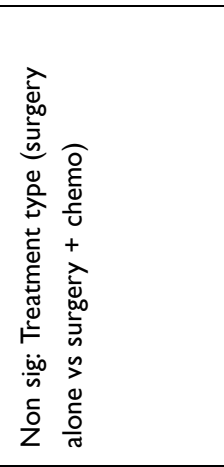 & & 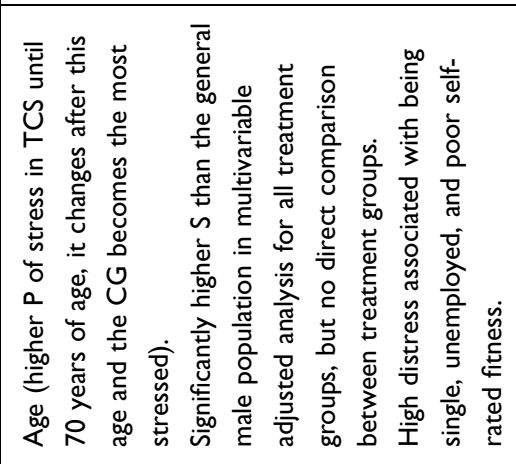 \\
\hline 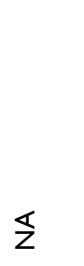 & 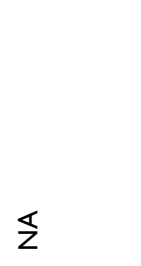 & z & 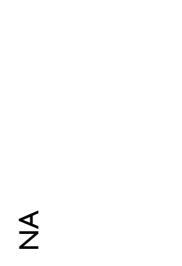 & $\S$ & & 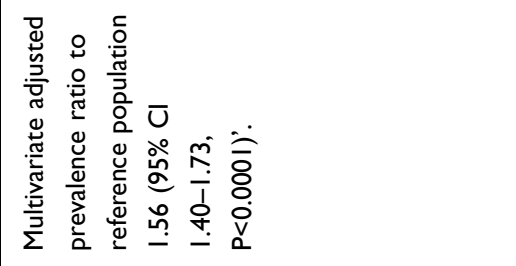 \\
\hline 兑 & 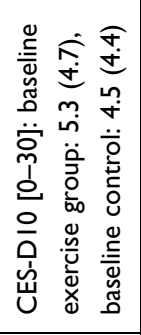 & 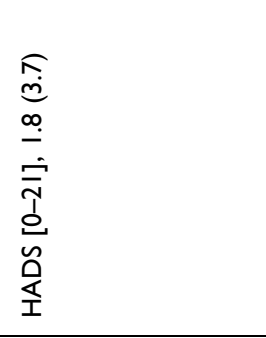 & 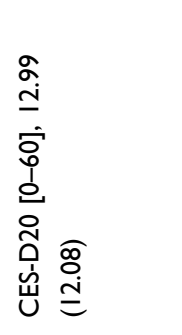 & 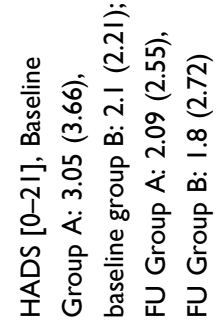 & & 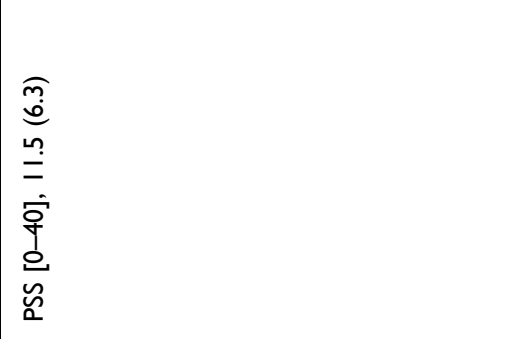 \\
\hline 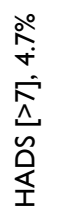 & $\frac{\alpha}{z}$ & 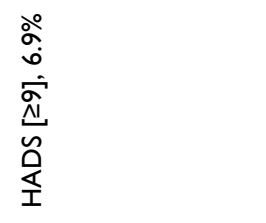 & $\stackrel{\alpha}{Z}$ & $\stackrel{\alpha}{Z}$ & & 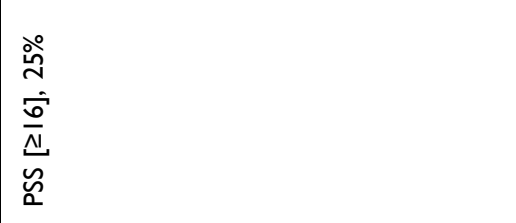 \\
\hline 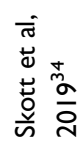 & 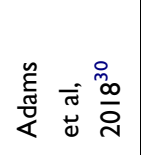 & 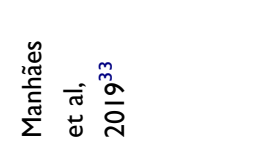 & 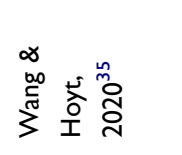 & 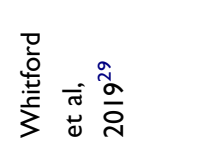 & & 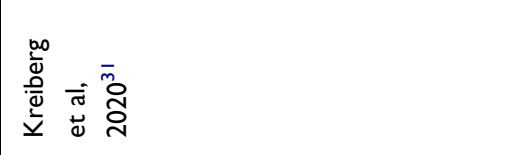 \\
\hline $\begin{array}{l}\text { Do } \\
\text { Oे }\end{array}$ & 唁 & 脑 & 脑 & 脑 & & $\begin{array}{l}8 \\
\circ \\
\end{array}$ \\
\hline$m$ & + & in & $r$ & $\infty$ & 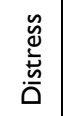 & $N$ \\
\hline
\end{tabular}




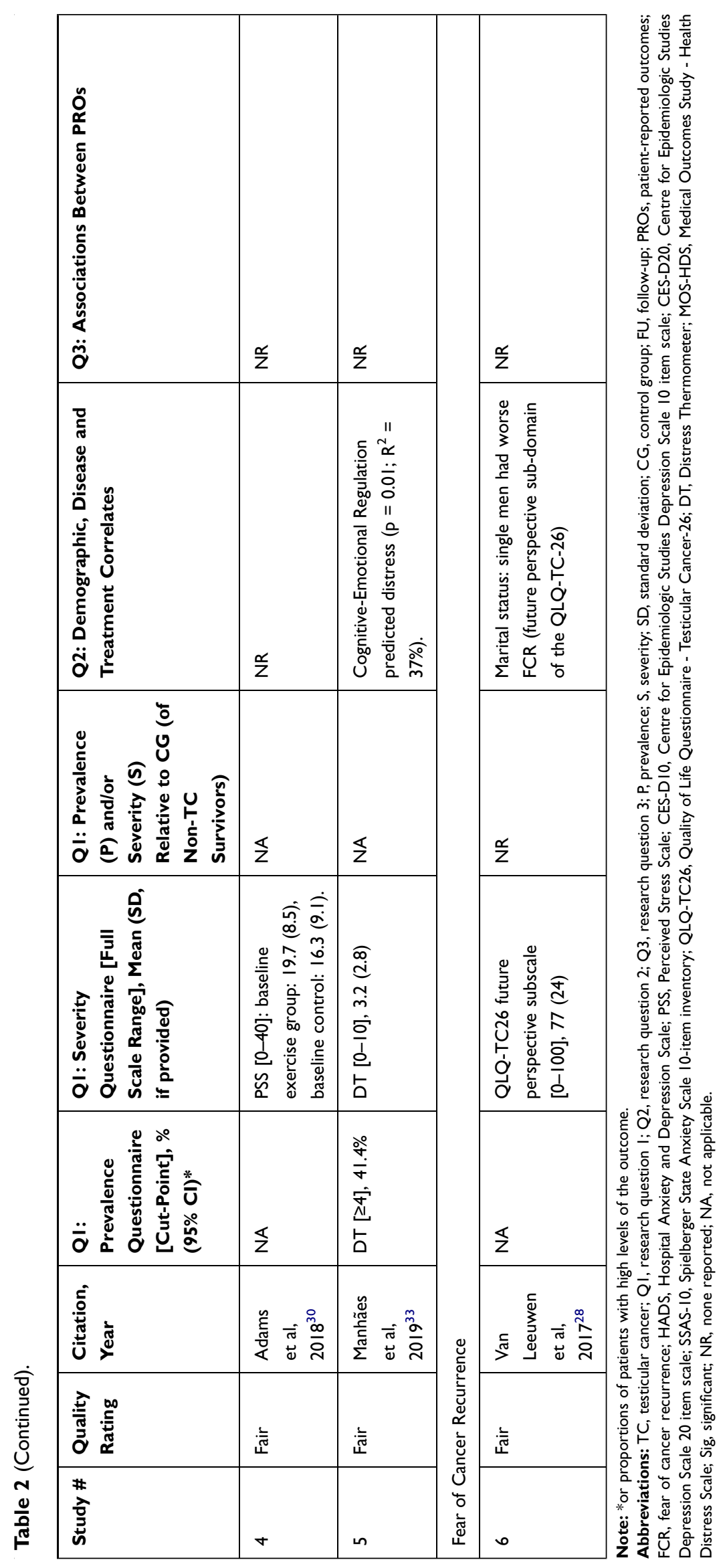




\section{Discussion}

The results of the current review suggest that anxiety is the main psychological burden for TC survivors, while depression, FCR and distress seem less prevalent and severe. Most ( 2 out of 3 ) studies reviewed found a similar prevalence of clinically significant anxiety (approximately 1 in 5) to our previous review. ${ }^{10}$ This is also broadly consistent with the prevalence of anxiety $(21 \%)$ reported in a systematic review of long-term ( $\geq 5$ years post-diagnosis) cancer survivors generally, ${ }^{36}$ but higher than prevalence estimates (eg, 9\%) from large cohorts. ${ }^{37}$ While there was no comparison with general population controls among studies in the current review, previous evidence suggests that anxiety is more prevalent in TC survivors than in the general population (estimated anxiety prevalence 1 in $8^{10}$ ). The studies that reported mean HADS-A scores were all of fair quality and HADS-A scores ranged from 3.2 to 6.16 , lower than the range of mean HADS-A scores reported in our previous review (4.1 to 4.8) and broader than HADSA score ranges for cancer survivors more generally $\left(3.8^{38}\right.$ to $5.5^{39}$ ). The small samples in most of the studies may account for these differences, and the new findings do not change the conclusion from our previous review that TC survivors experience elevated mean anxiety levels. TC survivors may experience greater anxiety due to the existential challenge to their sense of invulnerability posed by being diagnosed with a life-threatening illness at a relatively young age. They may also worry about dealing with common life challenges that young and middle-aged men already face (eg, establishing a family and career) in combination with the body image, sexuality and fertility issues that come with $\mathrm{TC}^{5-7}$

More efforts are needed to determine patient characteristics that increase the risk of higher anxiety scores. One small fair-quality study found anxiety was associated with sexual confidence and functioning, and cognitiveemotional regulation. These findings add to the emerging evidence of a link between anxiety and sexual problems and the consistent associations between anxiety and coping efforts identified in our previous review. Together with self-reported physical symptoms and side effects, these factors are the most well-established indicators of TC survivors at greater risk of anxiety. Further research is needed to identify factors associated with greater risk of anxiety, but given the limited number of consistent risk factors identified, screening TC survivors for anxiety and offering them options to reduce anxiety through self- management (eg, e-TC) or consultation with an allied health professional is recommended.

Consistent with our previous review, depression appears to burden TC survivors less so than anxiety. Fewer than 1 in 14 TC survivors were found to have clinically significant depression in the currently reviewed studies (based on 3 studies including for 2436 TC survivors). This is lower than the prevalence estimate ( 1 in 10) from the previous review, perhaps due to the use of different clinical cut-offs or cross-cultural variability, and lower than the prevalence of anxiety ( 1 in 5 ). Depression severity scores on both the HADS-D (1.8-3.05) and CESD (12.99) found in the current review were broadly consistent with those reported in our previous review and somewhat lower than what is seen in large mixed cohorts of cancer survivors (HADS-D M=5.0) and the general population (HADS-D M=5.4). ${ }^{39}$

The lower prevalence and severity of depression compared with anxiety found in studies of TC survivors may indicate that prototypic symptoms of depression, such as sadness or anhedonia, are less common in these men. Both qualitative $^{40}$ and quantitative ${ }^{41}$ studies have reported a renewed appreciation for life and a more positive outlook after TC. However, it may also be that commonly used measures of depression (eg, the HADS-D), do not assess externalizing depressive symptoms (eg, substance misuse, risk-taking, and poor impulse control) that are reported more often, and with greater intensity, in men versus women with depression. ${ }^{42}$ Future studies of TC survivors could incorporate measures, such as the Male Depression Risk Scale, ${ }^{43}$ that are more sensitive to these symptoms to ensure that the low burden of depression in TC survivors is not a measurement artefact.

Our previous review ${ }^{10}$ found that depression was associated with being unmarried/ lower social support, fatigue, negative health behaviours, avoidant and helpless/hopeless coping, poorer sexual functioning, previous psychological distress and altered body image and sense of masculinity. The current review identified further evidence supporting the relationship between depression and impaired masculinity, sexual functioning and coping strategies. As TC is a cancer of a sexual organ, concerns and challenges around meeting certain sexual and masculine stereotypes at a young age may cause symptoms of depression. The correlates of depression in TC survivors, such as threats to masculinity, sexual difficulties and maladaptive coping strategies, may not be immediately apparent or readily disclosed, screening for depression may be warranted. 
The prevalence of distress (approximately 1 in $3 \mathrm{TC}$ survivors) according to the two studies included in the current review is somewhat higher than the rate of 1 in 7 seen in our previous review. Both of these prevalence estimates sit within the range of $7 \%{ }^{36}$ to $46 \%{ }^{44}$ seen in recent reviews and cohort studies of cancer survivors generally. The variation is likely to be due to the different measures and cut-offs used to assess distress.

While distress seems more common in TC survivors than the general population, ${ }^{31}$ the current review produced little additional evidence regarding correlates of distress specifically related to the experience of TC. Further evidence was found for higher distress levels in TC survivors who are unemployed, unmarried (as in the general population) or with poor self-assessed fitness, but specific aspects of the diagnosis and treatment of TC (eg, treatment type) were unrelated to distress. Consistent correlates of distress identified in our previous review include self-reported cognitive complaints and side effects, and a passive coping style.

The current review found no new evidence regarding the prevalence of FCR in TC survivors, which is estimated to affect 1 in 3 men according to the previous review. ${ }^{10}$ One new study assessed FCR severity, and mean severity scores were found to be relatively low, adding to mixed evidence regarding the degree to which FCR affects TC survivors. Single/unmarried/un-partnered TC survivors were found to have higher FCR than partnered TC survivors. Generally, FCR has been found to be no higher in single versus partnered cancer survivors, ${ }^{45}$ but it may be more problematic for single TC survivors, as TC survivors perceive a greater threat to their future relationships and fertility from potential recurrence. ${ }^{40}$

The results of this review and our previous review suggest that anxiety is the most problematic psychological consequence of TC, although other issues such as depression may be under-recognized, and limited evidence is available regarding FCR. Our previous review found that clinical variables, such as treatment type and time since treatment, which may have offered a useful signpost for clinicians of men at risk of poorer outcomes, were largely unrelated to psychological outcomes; this review found no new evidence to suggest otherwise. Preliminary evidence suggests that factors influencing how men cope with the diagnosis and treatment of TC (eg, social support, coping style and sexual concerns) are more related to psychological outcomes in survivorship. Hence, it is not immediately apparent which TC survivors are at greatest risk of experiencing poorer psychological outcomes and when intervention may be most appropriate. While several large cross-sectional studies of TC survivors have been undertaken, well-powered theoretically driven longitudinal studies using validated psychological measures to explore changes in psychological outcomes over time are still needed. Techniques such as latent class analysis may help identify the combination of factors that differentiate the minority of TC survivors who experience poorer outcomes from the majority who adjust well. While it was outside the scope of this review, more research is also needed to understand how TC survivors' psychological outcomes impact on partners' or caregivers' well-being and ability to provide support. ${ }^{46}$ Better supporting TC survivors caregivers' may help optimise outcomes for both TC survivors and their caregivers, as greater social support has been associated with better psychological outcomes.

Given the lack of clear and consistent correlates of poorer psychological outcomes in TC survivors, psychosocial screening with a tool such as the Edmonton Symptom Assessment System (ESAS) ${ }^{47}$ or Distress Thermometer and problem list, ${ }^{48}$ ideally supplemented with a single item assessing FCR is recommended. ${ }^{49}$ Considering that many psychological outcomes appear to have little relationship with time since diagnosis or treatment, repeated screening at treatment initiation, completion and follow-up would be prudent.

There are a growing number of psychological interventions for TC survivors. ${ }^{22,25}$ In addition to focusing on the issues of greatest concern for TC survivors (ig anxiety and FCR) it is important that the ways in which these interventions address these issues are cognisant of masculine norms around help-seeking. A review of 37 men's depression helpseeking studies found that strength- and courage-based masculine norms could be used to promote help-seeking and that men prefer collaborative interventions involving action-oriented problem-solving. ${ }^{50}$ Sport-based approaches with embedded psychoeducation have been endorsed by TC survivors $^{51}$ and may have the added benefit of providing additional social support, which is associated with better outcomes.

The limitations of this updated review include the relative number of new studies found during the systematic search, which limited the likelihood of any different conclusions from our previous review. Another limitation was the variety of outcome measures utilized by the reviewed studies, which made synthesizing and 
interpreting results difficult. Unfortunately, many studies did not assess or report correlates of the psychological outcomes assessed, which hindered conclusions about those TC survivors likely to experience poorer outcomes.

\section{Conclusions}

This updated review of psychological outcomes in TC survivors provides additional evidence that a minority of TC survivors experience poor psychological outcomes, with anxiety being the most common issue. Men who are single or unemployed appear most at risk of poorer outcomes (particularly anxiety and depression). Greater anxiety and depression seems to be associated with impaired masculinity and sexual function, adding to the evidencebase for the relationship between poorer psychological outcomes and impaired QOL. More research is needed to identify TC survivors likely to experience poorer psychological outcomes and benefit from intervention.

\section{Acknowledgments}

This study did not receive any funding.

\section{Disclosure}

The authors report no conflicts of interest in this work.

\section{References}

1. Wild CPWE, Stewart BW, editors. World Cancer Report: Cancer Research for Cancer Prevention. Lyon, France: International Agency for Research on Cancer; 2020.

2. Bray F, Ferlay J, Soerjomataram I, Siegel RL, Torre LA, Jemal A. Global cancer statistics 2018: GLOBOCAN estimates of incidence and mortality worldwide for 36 cancers in 185 countries. CA Cancer J Clin. 2018;68(6):394-424. doi:10.3322/caac.21492

3. Fosså SD, Fung C, Dahl AA. Late adverse outcomes after treatment of testicular cancer. Onkologe. 2018;24:104-109. doi:10.1007/s00761018-0442-8

4. Cheng L, Albers P, Berney DM, et al. Testicular cancer. Nat Rev Dis Primers. 2018;4(1):1-24.

5. Alexis O, Adeleye AO, Worsley AJ. Men's experiences of surviving testicular cancer: an integrated literature review. J Cancer Surv. 2020;14(3):284-293. doi:10.1007/s11764-019-00841-2

6. Cappuccio F, Rossetti S, Cavaliere C, et al. Health-related quality of life and psychosocial implications in testicular cancer survivors. A literature review. Eur Rev Med Pharmacol Sci. 2018;22 (3):645-661. doi:10.26355/eurrev_201802_14290

7. Schepisi G, De Padova S, De Lisi D, et al. Psychosocial issues in long-term survivors of testicular cancer. Front Endocrinol. 2019;10:113. doi:10.3389/fendo.2019.00113

8. Ljungman L, Eriksson LE, Flynn KE, et al. Sexual dysfunction and reproductive concerns in young men diagnosed with testicular cancer: an Observational Study. J Sexual Med. 2019;16(7):1049-1059. doi:10.1016/j.jsxm.2019.05.005

9. Sztankay M, Giesinger JM, Holzner B. Quality of life and psychosocial burden in testicular cancer patients. Der Onkologe. 2017;23 (2):136-145. doi:10.1007/s00761-016-0158-6
10. Smith AB, Rutherford C, Butow P, et al. A systematic review of quantitative observational studies investigating psychological distress in testicular cancer survivors. Psychooncology. 2018;27 (4):1129-1137. doi:10.1002/pon.4596

11. Fleer J, Sleijfer D, Hoekstra H, Tuinman M, Klip E, HoekstraWeebers J. Objective and subjective predictors of cancer-related stress symptoms in testicular cancer survivors. Patient Educ Couns. 2006;64(1):142-150. doi:10.1016/j.pec.2005.12.009

12. Mykletun A, Dahl AA, Haaland CF, et al. Side effects and cancer-related stress determine quality of life in long-term survivors of testicular cancer. $J$ Clin Oncol. 2005;23(13):3061-3068. doi:10.1200/JCO.2005.08.048

13. Skaali T, Fosså SD, Andersson S, et al. Self-reported cognitive problems in testicular cancer patients: relation to neuropsychological performance, fatigue, and psychological distress. $J$ Psychosom Res. 2011;70(5):403-410. doi:10.1016/j.jpsychores.2010.12.004

14. Fosså SD, Dahl AA, Loge JH. Fatigue, anxiety, and depression in long-term survivors of testicular cancer. J Clin Oncol. 2003;21 (7):1249-1254. doi:10.1200/JCO.2003.08.163

15. Orre IJ, Fosså SD, Murison R, et al. Chronic cancer-related fatigue in long-term survivors of testicular cancer. J Psychosom Res. 2008;64 (4):363-371. doi:10.1016/j.jpsychores.2008.01.002

16. Allan S, Butow P, Olver I, et al. The prevalence, severity, and correlates of psychological distress and impaired health-related quality of life following treatment for testicular cancer: a survivorship study. J Cancer Surv. 2016;10(2):223-233. doi:10.1007/s11764-015-0468-5

17. Shinn EH, Basen-Engquist K, Thornton B, Spiess P, Pisters L. Health behaviors and depressive symptoms in testicular cancer survivors. Urology. 2007;69(4):748-753. doi:10.1016/j.urology.2006.12.022

18. Darabos K, Hoyt MA. Masculine norms about emotionality and social constraints in young and older adult men with cancer. J Behav Med. 2017;40(2):259-270. doi:10.1007/s10865-016-9739-5

19. Bumbasirevic U, Bojanic N, Pekmezovic T, et al. Health-related quality of life, depression, and sexual function in testicular cancer survivors in a developing country: a Serbian experience. Support Care Cancer. 2013;21(3):757-763. doi:10.1007/s00520-012-1577-6

20. Fung C, Dinh PC, Fossa SD, Travis LB. Testicular cancer survivorship. J Natl Compr Canc Netw. 2019;17(12):1557-1568. doi:10.6004/jnccn.2019.7369

21. Mora HDL, López C, Jimenez B, et al. Fear of recurrence as a triage strategy to identify testicular cancer survivors in need of psychological interventions. J Clin Oncol. 2018;36(15_suppl):e22110-e22110. doi:10.1200/JCO.2018.36.15_suppl.e222110

22. Smith AB, Rincones $\mathrm{O}$, Heiniger $\mathrm{L}$, et al. Online psychological self-management intervention for testicular cancer survivors (e-TC 2.0): promising acceptability and preliminary efficacy, but limited feasibility. J Psychosoc Oncol Res Pract. 2020;2(3):e33.

23. Vandraas KF, Reinertsen KV, Kiserud CE, Lie HC. Fear of cancer recurrence among young adult cancer survivors-exploring longterm contributing factors in a large, population-based cohort. J Cancer Surv. 2020. doi:10.1007/s11764-020-00943-2

24. Yang Y, Li W, Wen Y, et al. Fear of cancer recurrence in adolescent and young adult cancer survivors: a systematic review of the literature. Psychooncology. 2019;28(4):675-686. doi:10.1002/pon.5013

25. Hoyt MA, Wang AW-T, Ryan SJ, Breen EC, Cheavens JS, Nelson CJ. Goal-Focused Emotion-Regulation Therapy (GET) for young adult survivors of testicular cancer: a pilot randomized controlled trial of a biobehavioral intervention protocol. Trials. 2020;21(1):325. doi:10.1186/s13063-020-04242-0

26. Shamseer L, Moher D, Clarke M, et al. Preferred reporting items for systematic review and meta-analysis protocols (PRISMA-P) 2015: elaboration and explanation. BMJ. 2015;349.

27. Downs SH, Black N. The feasibility of creating a checklist for the assessment of the methodological quality both of randomised and non-randomised studies of health care interventions. J Epidemiol Community Health. 1998;52(6):377-384. doi:10.1136/jech.52.6.377 
28. van Leeuwen M, Kieffer JM, Efficace F, et al. International evaluation of the psychometrics of health-related quality of life questionnaires for use among long-term survivors of testicular and prostate cancer. Health Qual Life Outcomes. 2017;15(1):1. doi:10.1186/ s12955-017-0670-4

29. Whitford HS, Kalinowski P, Schembri A, et al. The impact of chemotherapy on cognitive function: a multicentre prospective cohort study in testicular cancer. Support Care Cancer. 2019. doi:10.1007/ s00520-019-05095-3

30. Adams SC, Delorey DS, Davenport MH, Fairey AS, North S, Courneya KS. Effects of high-intensity interval training on fatigue and quality of life in testicular cancer survivors. $\mathrm{Br} J$ Cancer. 2018;118(10):1313-1321. doi:10.1038/s41416-018-0044-7

31. Kreiberg M, Bandak M, Lauritsen J, et al. Psychological stress in long-term testicular cancer survivors: a Danish nationwide cohort study. J Cancer Surv. 2020;14(1):72-79. doi:10.1007/s11764-01900835-0

32. Bandak M, Lauritsen J, Johansen C, et al. Sexual function and quality of life in a national cohort of survivors of bilateral testicular cancer. Eur Urol Focus. 2018.

33. Manhães MFM, Okuma GY, Pedras RN, De Domenico EBL, Bergerot CD. Biopsychosocial needs of survivors of testicular cancer and evidence of validation of a brief scale. Estud Psicol. 2019;36.

34. Skøtt JW, Lauritsen J, Kreiberg M, Daugaard G, Bandak M. Quality of life in long-term testicular cancer survivors with compensated leydig cell dysfunction. Clin Genitourin Cancer. 2019;17(1):e65e71. doi:10.1016/j.clgc.2018.09.004

35. Wang AWT, Hoyt MA. Cancer-related masculinity threat in young adults with testicular cancer: the moderating role of benefit finding. Anxiety Stress Coping. 2020;33(2):207-215. doi:10.1080/ 10615806.2020.1713447

36. Brandenbarg D, Maass SWMC, Geerse OP, et al. A systematic review on the prevalence of symptoms of depression, anxiety and distress in long-term cancer survivors: implications for primary care. Eur J Cancer Care. 2019;28(3):e13086. doi:10.1111/ecc.13086

37. Götze H, Friedrich M, Taubenheim S, Dietz A, Lordick F, Mehnert A. Depression and anxiety in long-term survivors 5 and 10 years after cancer diagnosis. Support Care Cancer. 2020;28 (1):211-220. doi:10.1007/s00520-019-04805-1

38. Villoria E, Lara L. Assessment of a hospital anxiety and depression scale for cancer patients. Rev Med Chil. 2018;146(3):300-307. doi:10.4067/s0034-98872018000300300

39. Hinz A, Herzberg PY, Lordick F, et al. Age and gender differences in anxiety and depression in cancer patients compared with the general population. Eur J Cancer Care. 2019;28(5):e13129. doi:10.1111/ ecc. 13129
40. Matheson L, Boulton M, Lavender V, et al. Dismantling the present and future threats of testicular cancer: a grounded theory of positive and negative adjustment trajectories. J Cancer Surv. 2016;10 (1):194-205. doi:10.1007/s11764-015-0466-7

41. Fleer J, Hoekstra HJ, Sleijfer DT, Tuinman MA, Hoekstra-Weebers JE. The role of meaning in the prediction of psychosocial well-being of testicular cancer survivors. Qual Life Res. 2006;15(4):705-717. doi:10.1007/s11136-005-3569-1

42. Oliffe JL, Rossnagel E, Seidler ZE, Kealy D, Ogrodniczuk JS, Rice SM. Men's depression and suicide. Curr Psychiatry Rep. 2019;21(10):103. doi:10.1007/s11920-019-1088-y

43. Rice SM, Fallon BJ, Aucote HM, Möller-Leimkühler AM. Development and preliminary validation of the male depression risk scale: furthering the assessment of depression in men. $J$ Affect Disord. 2013;151(3):950-958. doi:10.1016/j.jad.2013.08.013

44. Carlson LE, Zelinski EL, Toivonen KI, et al. Prevalence of psychosocial distress in cancer patients across 55 North American cancer centers. J Psychosoc Oncol. 2019;37(1):5-21. doi:10.1080/ 07347332.2018.1521490

45. Simard S, Thewes B, Humphris G, et al. Fear of cancer recurrence in adult cancer survivors: a systematic review of quantitative studies. J Cancer Surv. 2013;7(3):300-322. doi:10.1007/s11764-013-0272-z

46. De Padova S, Casadei C, Berardi A, et al. Caregiver emotional burden in testicular cancer patients: from patient to caregiver support. Front. Endocrinol. 2019;10:318.

47. Hui D, Bruera E. The edmonton symptom assessment system 25 years later: past, present, and future developments. J Pain Symptom Manage. 2017;53(3):630-643. doi:10.1016/j.jpainsymman.20 16.10.370

48. Jacobsen PB, Donovan KA, Trask PC, et al. Screening for psychologic distress in ambulatory cancer patients: a multicenter evaluation of the distress thermometer. Cancer. 2005;103(7):1494-1502. doi:10.1002/cncr.20940

49. Smith AB. Integrating fear of cancer recurrence screening into routine care: opportunities and challenges. Psychooncology. 2020.

50. Seidler ZE, Dawes AJ, Rice SM, Oliffe JL, Dhillon HM. The role of masculinity in men's help-seeking for depression: a systematic review. Clinical Psychol Rev. 2016;49:106-118. doi:10.1016/j. cpr.2016.09.002

51. Petrella AR, Sabiston CM, O'Rourke RH, Santa Mina D, Matthew AG. Exploring the survivorship experiences and preferences for survivorship care following testicular cancer: a Mixed Methods Study. J Psychosoc Oncol Res Pract. 2020;2(4):e038.

\section{Publish your work in this journal}

Cancer Management and Research is an international, peer-reviewed open access journal focusing on cancer research and the optimal use of preventative and integrated treatment interventions to achieve improved outcomes, enhanced survival and quality of life for the cancer patient.
The manuscript management system is completely online and includes a very quick and fair peer-review system, which is all easy to use. Visit http://www.dovepress.com/testimonials.php to read real quotes from published authors. 